\title{
TATA INSTITUTE RADIOCARBON DATE LIST VIII
}

\section{P. AGRAWAL, S. K. GUPTA, and SHEELA KUSUMGAR}

Tata Institute of Fundamental Research, Bombay-5, India

Given below are the dates of archaeologic and Quaternary samples measured by the proportional counting of methane gas. Details of the techniques used were published earlier (Agrawal et al., 1965).

The half-life used is $5568 \mathrm{yr}$; the base year for converting dates on A.D./B.C. scale is 1950. Ninety-five per cent activity of the NBS oxalic acid is used as the modern standard.

General Comment:* for the first time, the crucial Pre-Harappa site of Amri (Period IB) (Casal, 1964) has been dated to ca. 2900 B.c. (TF-864). Nindovari damb, a site of Kulli affiliation, gives ca. 2050 B.c. (TF-862). Bagor, a newly discovered Neolithic site of Rajasthan, has given a very early date of ca. 3800 B.c. (TF-1007). The material used was charred bones. More samples from this site are under processing. Inamgaon, a Chalcolithic site, has been placed ca. 1350 в.c. (TF-922 and -924). A few samples from the old workings of copper and gold mines too have been dated for the first time. Summaries of excavations appear in Lal (1967-69).

In connection with our Quaternary Project, a large number of samples from raised beaches, borings from the swamps (Singh, 1967) and the continental shelf of the Arabian coast have been dated. Samples were collected in collaboration with Birbal Sahni Inst. of Palaeobotany, Lucknow, Deccan College, Poona and Natl. Inst. of Oceanog., Goa. As a result of this program, a number of Late Quaternary eustatic events on the west coast have been dated. Climatic and ecologic reconstructions based on pollen also have been dated for Rajasthan and Bengal.

\section{ACKNOWLEDGMENTS}

The authors thank Prof. D. Lal for guidance and S. V. Kerkar for assistance.

\section{SAMPLE DESCRIPTIONS}

I. ARCHAEOLOGIC SAMPLES

\section{Amri series, West Pakistan}

Amri (26 $6^{\circ} \mathrm{N}$ Lat, $68^{\circ} \mathrm{E} \mathrm{Long}$ ), Dist. Dadu, was excavated by J. M. Casal, Mus. Guimet, Paris, who subm. the samples. Rootlets were handpicked and $\mathrm{NaOH}$ pretreatment was given in both samples.

TF-863. Amri culture

$4485 \pm 110$

Charcoal, Field Id. Ai A, Layer 19.

TF-864. Amri culture

Charcoal, Field Id. Ai A10, Layer 28c.
2535 B.C.

$4710 \pm 110$ 2760 B.C.

\footnotetext{
* Dates based on half-life, $5730 \mathrm{yr}$.
} 
TF-1007. Bagor, India, Neolithic deposits

$5620 \pm 125$

Charred bones from Bagor (25 $22^{\prime} \mathrm{N}$ Lat, $74^{\circ} 23^{\prime} \mathrm{E}$ Long), Dist. Bhilwara, Trench EI, depth 1 m, Sample 4, Field No. BGR 1968-9/EI-4. Sample subm. by V. N. Mishra, Deccan College, Poona-6.

\section{Bandlamottu Hill series, Andhra Pradesh}

Bandlamottu Hill (16 $16^{\circ} 13^{\prime} \mathrm{N}$ Lat, $79^{\circ} 40^{\prime} \mathrm{E}$ Long), Dist. Guntur, from old mine workings. Samples subm. by D. B. Sikka, Agnigundala Copper-Lead Project, Bollapalli. $\mathrm{NaOH}$ pretreatment given to both samples.

\section{TF-805. Old copper workings}

Burnt wood, Sample $\mathrm{Ag} / \mathrm{B} / \mathrm{W} / 02$.

\section{TF-806. Old copper workings}

Wood, Sample $\mathrm{Ag} / \mathrm{B} / \mathrm{W} / 1$.

\section{Inamgaon series, Maharashtra}

Inamgaon ( $18^{\circ} 35^{\prime} \mathrm{N}$ Lat, $74^{\circ} 32^{\prime} \mathrm{E}$ Long), Dist. Poona. The site was excavated by H. D. Sankalia, Deccan College, Poona, who subm. the samples.

\section{TF-923. Chalcolithic culture}

$2890 \pm 170$

940 в.C.

Charcoal from Mound 1, Trench C2, Layer 5, depth $1.8 \mathrm{~m}$, Field No. 133. $\mathrm{NaOH}$ pretreatment was given.

\section{TF-924. Chalcolithic culture}

Charcoal from section-scrappings from 1st and 2nd floors, Layer 2, Field No. 139. NaOH pretreatment was given.

\section{TF-922. Chalcolithic culture} No. 63 .

Charcoal from Mound 1, Trench C3, Layer 5, depth $1.3 \mathrm{~m}$, Field

\section{Kalibangan series, Rajasthan}

The site of Kalibangan (29 $25^{\prime} \mathrm{N}$ Lat, $74^{\circ} 05^{\prime} \mathrm{E}$ Long), Dist. Sri Ganganagar, has yielded remains of Pre-Harappa and Harappa cultures. Excavations are being jointly conducted by B. B. Lal and B. K. Thapar. Samples subm. by B. B. Lal, Dir. Gen. Archaeol., New Delhi-11. All samples were pretreated with $\mathrm{NaOH}$.

\section{TF-942. Harappa culture \\ $4055 \pm 110$ \\ 2105 B.C.}

Charcoal from Trench KLB-2, Loc. XAI-QD4, Layer 12, depth 3.45 m, Field No. 1967-68/4/KLB-2. 
TF-946. Harappa culture

Wood charcoal from Trench KLB-2, Loc. ZNI, Qd1, Layer 7, depth 2.25 m, Field No. 1967-68/18/KLB-2.

\section{TF-947. Harappa culture}

Wood charcoal from Trench KLB-2, Loc G5, Qd3, Layer 34 , depth 5.2 m, Field No. 1967-68/21/KLB-2.

\section{TF-948. Harappa culture}

$3815 \pm 100$ 3.11 m, Field No. 1967-68/22/KLB-2.

\section{TF-396. Kayatha, India, Chalcolithic culture}

Charcoal from Kayatha (23 $30^{\prime} \mathrm{N}$ Lat, $76^{\circ} \mathrm{E}$ Long), Dist. Ujjain, Trench KTH-1, Layer 32, depth $8 \mathrm{~m}$, Field No. 5. NaOH pretreatment was given. Sample subm. by V. S. Wakankar, Vikram Univ., Ujjain.

\section{TF-879. Kolar Mine, India}

$$
1460 \pm 110
$$

Wood from Kolar mine ( $12^{\circ} 57^{\prime} \mathrm{N}$ Lat, $78^{\circ} 16^{\prime}$ E Long), Dist. Kolar, No. 2 Shaft area. Subm. by M.G.A. Mine Champion Reefs, P.O., KGF-3, Mysore.

\section{TF-759. Kotia, India, Late Quaternary}

$$
39.63 \pm 1.6 \%
$$

\section{Modern}

Caliche from Kotia (21 $50^{\prime} \mathrm{N}$ Lat, $73^{\circ} 15^{\prime} \mathrm{E}$ Long), Dist. Broach, from height $24.5 \mathrm{~m}$, from freshly exposed sec. of Narmada R. Subm. by K. T. M. Hegde to date river terraces. Comment: geochemistry of caliche not understood, hence "dates" expressed in percentage terms.

TF-966. Kulur, India, River sediments

Root of tree from Gurpur R. sediments, Dist. Mangalore, depth 13.7 m, Sample 2, Field 2. Sample subm. by E. Nielson. Comment: $\mathrm{NaOH}$ pretreatment was given. Sample dates a river bed sediment.

TF-822. Meja Dam, India

A.D. 1825

$125 \pm 90$

Wood from Meja Dam (24 $52^{\prime} \mathrm{N}$ Lat, $80^{\circ} 24^{\prime} \mathrm{E}$ Long), Dist. Mirzapur, depth $2 \mathrm{~m}$, Field No. MEJA/4. Subm. by V. S. Krishnaswamy, Geol. Survey of India, Lucknow. $\mathrm{NaOH}$ pretreatment was given.

\section{TF-862. Nindowari damb, West Pakistan, Kulli culture \\ $3900 \pm 105$ \\ 1950 B.C.}

Charcoal from Nindowari (27 $\mathrm{N}$ Lat, $66^{\circ} 30^{\prime} \mathrm{E}$ Long), Dist. Khuzdar, from Trench ND, Layer 3, Field Id. ND. B1-XXIV. Site was ex- 
cavated by J. M. Casal who subm. the sample. $\mathrm{NaOH}$ pretreatment was given.

\section{Paiyampalli series, Madras}

Paiyampalli $\left(12^{\circ} 33^{\prime} \mathrm{N}\right.$ Lat, $78^{\circ} 25^{\prime}$ E Long), Dist. North Arcot; samples subm. by B. B. Lal.

\section{TF-823. Megalithic}

$2515 \pm 100$

Charred grains and charcoal pieces in a pit on Floor 3, Trench B1, Loc. Qd2, Layer 4, depth $1.7 \mathrm{~m}$, Field No. PMP/4. NaOH pretreatment was given.

TF-824. Megalithic (?)

$785 \pm 90$

Charcoal from Trench A2, Pit 6 sealed by Layer 4, depth $1.21 \mathrm{~m}$, Field No. PMP/8. NaOH pretreatment was given.

\section{TF-825. Megalithic (?)}

$695 \pm 95$

Charcoal from Trench A2, Pit 1 sealed by Layer 5, depth $0.9 \mathrm{~m}$, Field No. PMP/8. NaOH pretreatment was given.

TF-827. Neolithic (?)

$3570 \pm 105$

1620 в.c.

Charcoal from Trench Al, Pit 3 sealed by Layer 6A, depth $2 \mathrm{~m}$, Field No. PMP/8. NaOH pretreatment was given.

\section{TF-828. Megalithic}

$$
2100 \pm 95
$$

150 B.c.

Charcoal from Trench A1, Layer 6A, depth 1.7 m, Field No. PMP/8. $\mathrm{NaOH}$ pretreatment was given.

\section{TF-829. Neolithic (?)}

Charcoal from Trench A2, Pit 9 sealed by Layer 7, depth $1.9 \mathrm{~m}$, Field No. PMP/8. NaOH pretreatment was given.

TF-832. Neolithic (?)

$770 \pm 100$

Charcoal from Trench Al, Layer 8, depth 1.9 m, Field No. PMP/8.

TF-833. Neolithic (?)

$3215 \pm 210$

1265 B.C.

Charcoal from Tr. XF1, Qdt. 2, Layer 8, depth 1.9 m, Field No. PMP/8. Comment: as the sample was small, anthracite was mixed for counting.

General Comment: considerable scatter shown by the $\mathrm{C}^{14}$ dates cannot be explained by contamination. A more controlled sampling of the site is indicated.

\section{Palavoy series, Andhra Pradesh}

Palavoy (14 $31^{\prime} \mathrm{N}$ Lat, $77^{\circ} 09^{\prime} \mathrm{E}$ Long), Dist. Ananthpur. Samples subm. by H. D. Sankalia. 
TF-699. Ashmound

Modern sample.

Carbonaceous clay from Layer 2. Comment: iron slag was found with

TF-700. Neolithic 1440 B.c.

$3390 \pm 95$

Carbonaceous ash (dung) from Layer 7, depth $2.1 \mathrm{~m}$, sample No. 2.

\section{B.c.}

Charcoal from Pirak (29 $30^{\prime} \mathrm{N}$ Lat, $67^{\circ} 54^{\prime}$ E Long), Dist. Kanchi, Layer 12, depth $1 \mathrm{~m}$, Field PK.A. Site excavated by J. M. Casal who subm. sample. Comment: date agrees with Casal's estimate. NaOH pretreatment was given.

\section{TF-921. Sonkh, India, Early historic deposits}

\section{$10,095 \pm 300$
8145 в.C.}

Wood from Spirit Cave $\left(20^{\circ} \mathrm{N}\right.$ Lat, $98^{\circ} \mathrm{E}$ Long), Dist. Prov. Hongson, Loc. B3, Layer 3, depth 0.3 m, Field B3 (3). Sample subm. by C. F. Gorman, Archaeol. Lab., Hawaii, Honolulu.

II. QUATERNARY SAMPLES

TF-907. Aramra, Late Quaternary

Dead coral from surface near village of Aramra $\left(22^{\circ} 26^{\prime} \mathrm{N}\right.$ Lat, $69^{\circ} 05^{\prime}$ E Long), Dist. Jamnagar, Field 24. Coll. by S. K. Gupta.

\section{TF-905(a). Bardia village, India, Late Quaternary}

$14,565 \pm 185$

Shells from Bardia village $\left(22^{\circ} 11^{\prime} \mathrm{N}\right.$ Lat, $69^{\circ} 02^{\prime} \mathrm{E}$ Long). Dist. Jamnagar, from depth $2 \mathrm{~m}$, Field Loc. 29. Coll. by S. K. Gupta.

\section{TF-908. Bhimrana village, Late Quaternary}

$5275 \pm 105$ 3325 B.C.

Shells from Bhimrana village $\left(22^{\circ} 23^{\prime} \mathrm{N}\right.$ Lat, $69^{\circ} 02^{\prime} \mathrm{E}$ Long), Dist. Jamnagar, from raised beach, depth $1.25 \mathrm{~m}$, Field Loc. 29. Coll. by S. K. Gupta.

General Comment: samples date eustatic changes as recorded by Kathiawar peninsula.

TT-969. Off Bombay, Indlia, continental shelf

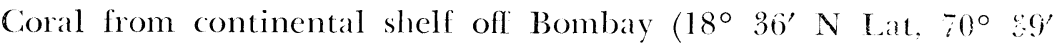
fi Long), depth $96 \mathrm{~m}$, Field 4?(b). Sample subm. by R. R. Nair, Natl. 
Inst. Oceanog., Panaji, Goa. Comment: for studying Quaternary sealevel changes.

TF-814. Coondapoor town, India

Submerged mangrove plants from Coondapoor $\left(13^{\circ} 30^{\prime} \mathrm{N}\right.$ Lat, $74^{\circ}$ 4' E Long), Dist. S Kanara. Subm. by K. S. Karanth, Puttur, S Kanara. $\mathrm{NaOH}$ pretreatment was given. Comment: sample dates a marine transgression.

\section{TF-897(b). Dhrubya Hill, India Late Quaternary $\quad 10,330$ в.c.}

Miliola tests from Dhrubya Hill, Dist. Kutch, from surface, Field 11/78. Coll. and subm. by S. K. Biswas, Oil Nat. Gas. Comm., Baroda.

\section{Erangal-Bhatti series, Maharashtra}

Erangal-Bhatti ( $18^{\circ} 36^{\prime} \mathrm{N}$ Lat, $70^{\circ} 39^{\prime} \mathrm{E}$ Long), Dist. Bombay. Samples coll. by D. P. Agrawal from raised beach.

TF-981. Late Quaternary

$4925 \pm 100$

Shells from depth $2.9 \mathrm{~m}$, Sample 6, Field Pit 1 . 2975 B.C.

\section{TF-972. Late Quaternary}

Shells from depth $0.6 \mathrm{~m}$, Sample 2, Field 2/Pit 1.

705 B.C.

TF-938. Late Quaternary

$1715 \pm 95$

Shells from depth $0.8 \mathrm{~m}$, Sample 3, Field Madh/2.

General Comment: above samples consist of consolidated comminuted shells from raised beach, which represents a Holocene transgression. TF-972 and -938 indicate some stratigraphic disturbance.

\section{TF-915. Jhinjunvada, India, Late Quaternary}

$6640 \pm 125$

Shells from Jhinjunvada (23 $24^{\prime} \mathrm{N}$ Lat, $71^{\circ} 32^{\prime} \mathrm{E}$ Long), Dist. Surendra Nagar, from a brine well, depth 5.1 to $5.5 \mathrm{~m}$. Sample coll. by S. K. Gupta. Comment: sample dates a Holocene regression in Little Rann of Kutch.

TF-898. Jura Hill, India, Late Quaternary $-885$

Miliola tests from surface of Jura Hill, Dist. Kutch, Field 11/61. Coll. and subm. by S. K. Biswas. Comment: sample dates miliolite formations of Gujarat.

\section{TF-983. Continental shelf, off Karwar, India}

$$
8880 \pm 125
$$

Mollusc shells from continental shelf off Karwar $\left(10^{\circ} 33^{\prime} \mathrm{N}\right.$ Lat, $73^{\circ} 13^{\prime} \mathrm{E}$ Long), depth below water surface $58.5 \mathrm{~m}$, Sample 653. Sample subm. by R. R. Nair. Comment: sample dates a eustatic event. 


\section{Katral Hill series, Gujarat}

Katral Hill, Dist. Kutch. Samples subm. by S. K. Biswas to date Kutch miliolite formations.

TF-893. Late Quaternary

Miliola shells from surface, Field 11/26.

TF-892. Late Quaternary

Miliola shells from depth $7.6 \mathrm{~m}$, Field 11/92.
$+1600$ 26,645 в.C.

32,530

$+2710$

30,580 в.c.

\section{Kharagodha series, India}

Kharagodha $\left(23^{\circ} 10^{\prime} \mathrm{N}\right.$ Lat, $71^{\circ} 39^{\prime} \mathrm{E}$ Long), Dist. Surendra Nagar. Samples coll. by S. K. Gupta from a brine well. Samples date Holocene marine regressions in Little Rann of Kutch.

\section{TF-917. Late Quaternary $\quad 6835 \pm 110$}

Wood from Damod, depth 5.4 to $6.1 \mathrm{~m}$, Loc. 2. $\mathrm{NaOH}$ pretreatment was given.

\section{TF-919. Late Quaternary}

Shells from depth 2.6 to $2.9 \mathrm{~m}$, Loc. 2 .

$5900 \pm 105$

TF-920. Late Quaternary 3950 B.C. girch

$6860 \pm 110$

4910 в.C.

Wood from depth 2.4 to $3.5 \mathrm{~m}$, Loc. 1 . NaOH pretreatment was

\section{Kuda series, Gujarat}

Kuda $\left(23^{\circ} 13^{\prime}\right.$ N Lat, $71^{\circ} 23^{\prime}$ E Long), Dist. Surendra Nagar. Samples coll. from brine well by S. K. Gupta to date marine regressions in Little Rann of Kutch.

\section{TF-913. Late Quaternary}

$6315 \pm 95$

Shells from depth $5.7 \mathrm{~m}$, Loc. 17.

TF-914. Late Quaternary 4365 B.C.

Shells from depth 7.9 to $8.2 \mathrm{~m}$, Loc. 16 .

\section{Minicoy Island series}

Minicoy I. ( $8^{\circ} 18^{\prime} \mathrm{N}$ Lat, $73^{\circ} \mathrm{E}$ Long). Samples coll. by S. G. Patil, Tata Inst. of Fundamental Research, Bombay, to date exposed coral
reefs. 
TF-1017. Exposed corals

$1575 \pm 85$

Coral, pure aragonite, from depth $3 \mathrm{~m}$. Sample 2, Field A5.

TF-1022. Exposed corals

Modern

Coral, pure aragonite from depth $0.9 \mathrm{~m}$. Sample 3, Field All.

\section{Nicora series, Gujarat}

Nicora $\left(21^{\circ} 46^{\prime} \mathrm{N}\right.$ Lat, $73^{\circ} 7^{\prime}$ E Long), Dist. Broach. Samples coll. and subm. by K. T. M. Hegde, M.S. Univ., Baroda, to date river sediments by using caliche deposits. Comment: same as for TF-759.

TF-900. Late Quaternary

$12.31 \pm 0.34 \%$

Caliche coll. from Narmada R. bank. Sample 2.

Modern

TF-901. Late Quaternary

$10.90 \pm 0.36 \%$

Modern

Caliche coll. from Narmada R. bank. Sample 3.

TF-906. Okha, India, Late Quaternary

Coral from Okha $\left(22^{\circ} 28^{\prime} \mathrm{N}\right.$ Lat, $69^{\circ} 06^{\prime} \mathrm{E}$ Long), Dist. Jamnagar, Field 27. Coll. by S. K. Gupta, to date an emerged reef.

\section{TF-891. Paithan, India, Late Quaternary}

$18,490 \pm 650$

16,540 в.c.

Fresh-water shells from Paithan $\left(19^{\circ} 31^{\prime} \mathrm{N}\right.$ Lat, $75^{\circ} 22^{\prime} \mathrm{E}$ Long), Dist. Aurangabad, from an old floodplain of Godavari R., depth $5 \mathrm{~m}$. Subm. by A. Parthasarthy, Indian Inst. of Technol., Powai, Bombay.

\section{TF-911. Salaya, India, Late Quaternary}

$5075 \pm 105$

Dead coral from Salaya $\left(22^{\circ} 22^{\prime} \mathrm{N}\right.$ Lat, $69^{\circ} 39^{\prime} \mathrm{E}$ Long), Dist. Jamnagar. Sample coll. from well dug in the sea floor, Loc. 20. Coll. by S. K. Gupta.

\section{Sambhar Lake series, Rajasthan}

Sambhar Salt lake $\left(26^{\circ} 54^{\prime} \mathrm{N}\right.$ Lat, $75^{\circ} 13^{\prime}$ E Long), Dist. Jaipur. Coll. by G. Singh, Birbal Sahni Inst. of Palaeobotany, Lucknow, for pollen analysis and $\mathrm{C}^{14}$ dating. $\mathrm{NaOH}$ pretreatment given to all samples.

TF-883. Late Quaternary

$4385 \pm 110$

Organic debris from depth 1.3 to $1.5 \mathrm{~m}$, Field S2/135-150, Sample RC-6.

TF-884. Late Quaternary

$6060 \pm 105$

Organic debris from depth $1.9 \mathrm{~m}$, Field S2/185-195. Sample RC-7. 
TF-886. Late Quaternary

Organic debris from depth $2.9 \mathrm{~m}$, Field S2/285-295. Sample RC-9.

TF-887. Late Quaternary

$8990 \pm 125$

7040 B.c.

Organic debris from depth $3.2 \mathrm{~m}$, Field S2/315-325. Sample RC-10. General Comment: samples date wet and dry phases on the basis of pollen zones which indicate that Sambhar was a fresh water lake before 4000 B.P.

\section{Sankrail series, West Bengal}

Sankrail (22० $35^{\prime} \mathrm{N}$ Lat, $88^{\circ} 20^{\prime}$ E Long), Dist. Howrah, coll. and subm. by Vishnu-Mittre and H. P. Gupta, Birbal Sahni Inst. of Palaeobotany, Lucknow.

\section{TF-850. Late Quaternary}

$2540 \pm 100$

Peaty clay from depth $1.4 \mathrm{~m}$, Sample 1 .

590 B.c.

TF-851. Late Quaternary

$3960 \pm 95$

Peat from depth $1.8 \mathrm{~m}$, Sample 2.

TF-853. Late Quaternary

2010 B.C.

Wood from depth $1.5 \mathrm{~m}$, Sample 4 . $\mathrm{NaOH}$ pretreatment was given.

TF-855. Late Quaternary

Peat from depth $3 \mathrm{~m}$. Sample 6 .

TF-856. Late Quaternary

Peat from depth $6 \mathrm{~m}$, Sample 7 .

TF-857. Late Quaternary

Wood, depth not given. Sample 8.
$4785 \pm 105$

2835 B.C.

General Comment: samples date a pollen sequence and thus help ecologic and climatic reconstructions for Holocene in Bengal.

\section{Saurashtra coast series, Gujarat}

Saurashtra coast, samples coll. and subm. by M.V.A. Sastry, Geol. Survey of India, Calcutta, to date emerged coral reefs for eustatic studies.

TF-1015. Sub-Recent/Recent

Coral, pure aragonite. Sample 2, Field C.R. 2.

TF-1014. Sub-Recent/Recent

Coral aragonite. Sample 1, Field C.R. 1.
$4590 \pm 130$

2640 B.C.

$5645 \pm 105$

3695 в.C.

$5285 \pm 110$

3335 B.C. 


\section{Surajbari series, Gujarat}

Surajbari (23 $8^{\prime} \mathrm{N}$ Lat, $70^{\circ} 42^{\prime} \mathrm{E}$ Long), Dist. Malia, in Little Rann of Kutch. Samples coll. and subm. by S. K. Gupta, to date marine regressions.

TF-930. Late Quaternary

$3720 \pm 100$

Shells from depth $4.9 \mathrm{~m}$. Sample 3, Field RH 27(c)/3.

TF-932. Late Quaternary

1770 B.C.

Shells from depth $16 \mathrm{~m}$. Sample 7, Field RH 24/7.

$6600 \pm 105$

4650 B.c.

TF-927. Late Quaternary

$4685 \pm 100$

Shells from depth $7 \mathrm{~m}$. Sample 3, Field RH 27(d)/3.

TF-765. Takaopa, Thailand, Late Pleistocene

$>40,000$

Lignitic clay, from Takaopa ( $8^{\circ} 8^{\prime} \mathrm{N}$ Lat, $98^{\circ} 4^{\prime} \mathrm{E}$ Long), alluvial tin-mine area, depth $9 \mathrm{~m}$. Sample coll. by P. Aranyaknon, Royal Dept. of Mines, Bangkok, Thailand.

TF-903. Visavara village, India, Late Quaternary

25,760 в.c.

Coral from Visavara village $\left(21^{\circ} 45^{\prime} \mathrm{N}\right.$ Lat, $69^{\circ} 26^{\prime} \mathrm{E}$ Long), Dist. Junagadh, depth $0.3 \mathrm{~m}$, Loc. 32. Coll. by S. K. Gupta to date emerged reef.

\section{TF-889(a). Washtana, India, Late Quaternary}

$11,130 \pm 150$

Miliolite from Washtana $\left(23^{\circ} 25^{\prime} \mathrm{N}\right.$ Lat, $70^{\circ} 34^{\prime} \mathrm{E}$ Long), Dist. Waga, Field 11/132. Subm. by S. K. Biswas to date local miliolite formations.

\section{TF-965. Willington Island, India, $\quad 8080 \pm 120$ Postglacial sediments \\ 6130 B.C.}

Root of tree from Willington I., Dist. Cochin, depth $16.75 \mathrm{~m}$. Sample 1, Field 1. Sample subm. by E. Nielson, Cochin Port Trust. Comment: sample from postglacial marine or backwater sediments. $\mathrm{NaOH}$ pretreatment was given.

\section{REFERENCES}

Agrawal, D. P., Kusumgar, Sheela, and Lal, D., 1965, The measurement of $\mathrm{C}^{14}$ activity and some age determinations of archaeological samples: Current Sci. [India], v. 34, p. 394-397.

Casal, J. M., 1964, Fouilles d'Amri: Paris, Musee Guimet, 2 v.

Lal, B. B., 1967-1969, Indian archaeology-a review: Archaeol. Survey of India.

Singh, G., 1967, A palynological approach towards the resolution of some important desert_problems in Rajasthan: Indian Geohydrology, v. 3, no. 1, p. 11-128. 